\title{
In Situ TEM Investigation of the Spontaneous Hollowing of Alloy Anode Nanocrystals
}

Matthew Boebinger ${ }^{1}$, Olesya Yarema ${ }^{2}$, Maksym Yarema ${ }^{2}$, Kinga Unocic ${ }^{1}$, Raymond Unocic ${ }^{1}$, Vanessa Wood ${ }^{2}$ and Matthew McDowell ${ }^{3}$

${ }^{1}$ Oak Ridge National Laboratory, United States, ${ }^{2}$ ETH Zurich, United States, ${ }^{3}$ Georgia Institute of Technology, United States

Developing anode materials with a higher specific capacity has been an ongoing research priority to replace traditional graphitic anodes in Li-ion batteries (LIBs) [1,2]. Alloying of promising anode materials will allow more $\mathrm{Li}$ to be stored as opposed to intercalation-based anode chemistries; however, drastic volume and structural changes associated with these alloying reactions limit LIB cyclability and durability [3, 4]. These problems have previously been mitigated by using hollow and yolk-shell nanostructures with a dimensionally stable outer surface and a sufficiently large interior space that allows for the expansion of the active material during cycling $[4,5]$. This stable outer surface is key for increasing the stability of these nanomaterials as it allows for the passivating growth of the solid-electrolyte interphase (SEI); however, these architectures are very complex and can be expensive to synthesize [4]. In this study, in situ transmission electron microscopy (TEM) biasing experiments show that during lithiation, sufficiently small antimony nanocrystals (Sb NCs), $\sim 15 \mathrm{~nm}$ in diameter [6], form alloy NCs and during lithium removal spontaneously form hollow structures that can be reversibly filled during repeated LIB cycling [7]. Figure 1a shows how the hollow structures develop due to the presence of a resilient oxide layer that forms naturally on the surface of the Sb NCs after synthesis. In situ TEM images (Figures $1 \mathrm{~b}-1 \mathrm{e}$ ) show the lithiation/delithiation process - a $\mathrm{Li}_{3} \mathrm{Sb}$ alloy forms during lithiation and during lithium removal from the interior active $\mathrm{Li}_{3} \mathrm{Sb}$ alloy, the oxide surface layer mechanically prevents the shrinkage that typically occurs for such alloying materials. This enables delithiation to proceed via a different pathway, resulting in the formation of internal voids, as shown in Figure 1d.

A chemomechanical model (Figure 2) was developed to explain observations from the in situ TEM experiments and show that void formation is theoretically an energy tradeoff between the formation of the void's surface area and the stress that develops within the oxide surface layer. Void behavior is size dependent; the larger a particle grows, the lower the buckling stress needed for the oxide layer to fail, which results in particle shrinkage rather than void formation. The creation of other types of shells can be developed using this model as a guide to enable the use of high-capacity alloying materials by inducing similar hollowing transformations. A variety of electrochemical studies were performed to verify that the void behavior observed during in situ TEM experiments occurred in traditional LIB cells and also improved the overall electrochemical performance. Anodes comprised of small Sb NCs were found to consistently have a higher Coulombic efficiency compared to anodes prepared using larger Sb NCs. The ability for optimally sized Sb NCs to form hollow/alloy nanostructures, show that Sb can be a promising material for further study and use in nextgeneration, high-energy batteries with longer cycle life [8]. 


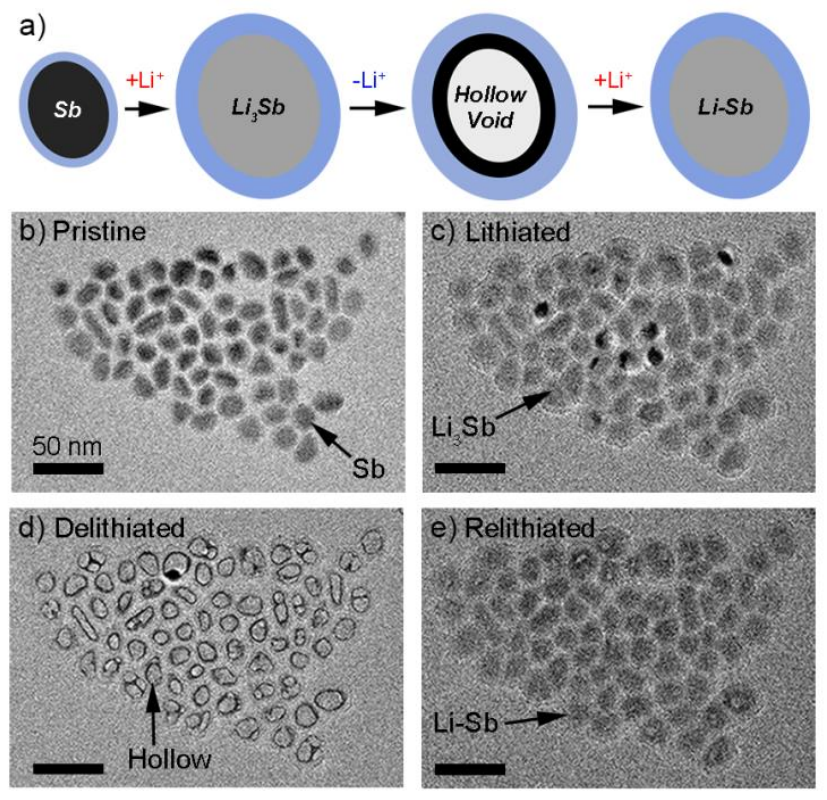

Figure 1. (a) Schematic representation of the lithiation/delithiation cycle observed experimentally in b-e. (be) In situ TEM sequence of Sb NCs as they undergo a full lithiation cycle and one relithiation [7].
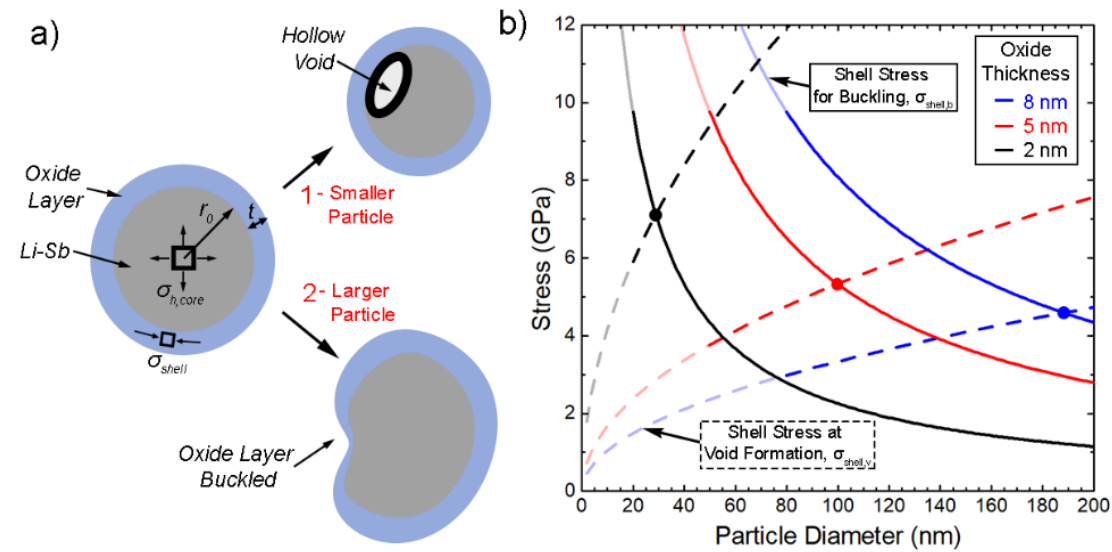

Figure 2. (a) Schematic of two reaction pathways lithiated Sb NCs undergo during delithiation and dependence on particle size, oxide shell thickness, and stress evolution in the core and oxide shell. (b) Plot of stress in shell at formed void (dotted lines) and shell stress for buckling (solid lines) vs. particle diameter with different oxide thicknesses. Below the crossover point, void formation is favored for that oxide thickness while above this point, oxide shell will buckle [7].

\section{References}

[1] Nitta, N., et al., Materials Today 18 (2015) p. 252

[2] Obrovac, M.N. and V.L. Chevrier, Chemical Reviews 114 (2014) p. 11444

[3] McDowell, M.T., S. Xia, and T. Zhu, Extreme Mechanics Letters 9 (2016) p. 480

[4] Liu, N., et al., Nature Nanotechnology 9 (2014) p. 187

[5] Liu, N., et al., Nano Letters 12 (2012) p. 3315

[6] He, M., et al., Nano Letters 14 (2014) p. 1255

[7] Boebinger, M.G., et al., Nature Nanotechnology 15 (2020) p. 475

[8] This work was performed at the Georgia Tech Materials Characterization Facilty and the Institute for Electronics and Nanotechnology, which is supported by the National Science Foundation. M.G.B. acknowledges support from the DOE Office of Science Graduate Student Research Program for research performed at Oak Ridge National Laboratory. A portion of this research was conducted at the Center for Nanophase Materials Sciences, which is a DOE Office of Science User Facility. 